\title{
A Single Cell Dissociation Approach for Molecular Analysis of Urinary Bladder in the Mouse Following Spinal Cord Injury
}

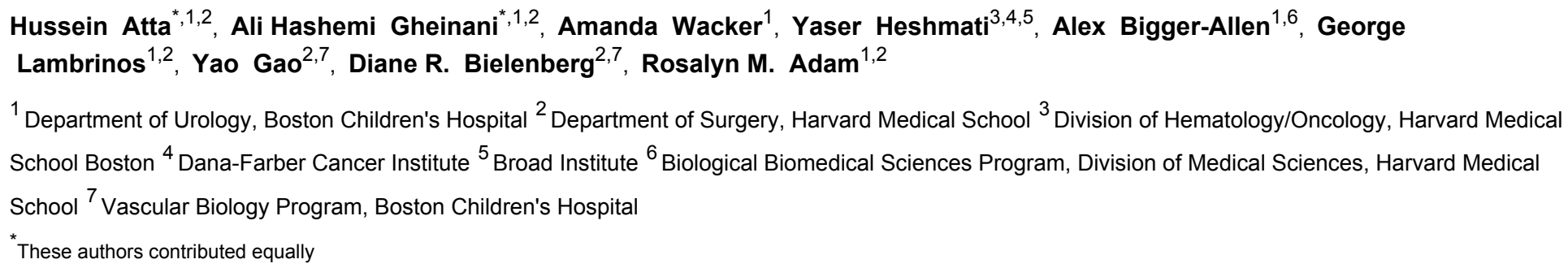

\section{Corresponding Authors}

Ali Hashemi Gheinani

Ali.HashemiGheinani@childrens.harvard.edu

Rosalyn M. Adam

Rosalyn.Adam@childrens.harvard.edu

\section{Citation}

Atta, H., Gheinani, A.H.,

Wacker, A., Heshmati, Y., Bigger-

Allen, A., Lambrinos, G., Gao, Y.

Bielenberg, D.R., Adam, R.M. A Single

Cell Dissociation Approach for Molecular

Analysis of Urinary Bladder in the Mouse

Following Spinal Cord Injury. J. Vis.

Exp. (160), e61455, doi:10.3791/61455

(2020).

\section{Date Published}

June 17, 2020

DOI

$10.3791 / 61455$

URL

jove.com/video/61455

\section{Abstract}

We describe the implementation of spinal cord injury in mice to elicit detrusor-sphincter dyssynergia, a functional bladder outlet obstruction, and subsequent bladder wall remodeling. To facilitate assessment of the cellular composition of the bladder wall in non-injured control and spinal cord injured mice, we developed an optimized dissociation protocol that supports high cell viability and enables the detection of discrete subpopulations by flow cytometry.

Spinal cord injury is created by complete transection of the thoracic spinal cord. At the time of tissue harvest, the animal is perfused with phosphate-buffered saline under deep anesthesia and bladders are harvested into Tyrode's buffer. Tissues are minced prior to incubation in digestion buffer that has been optimized based on the collagen content of mouse bladder as determined by interrogation of publicly available gene expression databases. Following generation of a single cell suspension, material is analyzed by flow cytometry for assessment of cell viability, cell number and specific subpopulations. We demonstrate that the method yields cell populations with greater than $90 \%$ viability, and robust representation of cells of mesenchymal and epithelial origin. This method will enable accurate downstream analysis of discrete cell types in mouse bladder and potentially other organs.

\section{Introduction}

Perturbations of normal urinary bladder function can lead to decreased quality of life for many individuals. In order to gain a better understanding of how injury or disease derails normal bladder function, it is important to probe 
the normal biological state of cells within the bladder and how they change under experimental perturbation. To date, however, the specific cell populations that reside within the urinary bladder, and how they change with injury, have been incompletely characterized.

Single cell profiling methods such as flow cytometry or single cell RNA sequencing (scRNA-seq) have the potential to shed light on specific cell types within the bladder. However, for these approaches to be informative tissue must be digested in a manner that does not affect viability, gene expression, and representative cell population percentages of the harvested tissue. Protocols that employ enzymatic disaggregation can impact surface marker expression through indiscriminate protease activity ${ }^{1}$, thereby impacting cell identification by flow cytometry, whereas the dissociation process itself can lead to the induction of immediate early genes, as described recently by Van den Brink and colleagues ${ }^{2}$. The authors showed that although the dissociation-affected subpopulation was small, it could trigger a strong contaminating signal in bulk expression studies due to the high expression levels of immediate early genes. In addition, the duration of the dissociation protocol affected detected bulk expression levels of genes shown to be unique to some subpopulations. Thus, single cell datasets generated without accounting for the impact of the dissociation protocol may yield gene expression changes arising from the dissociation method, as opposed to underlying biology. These observations suggest that published single cell transcriptomics data should be interpreted with caution, and that results should be validated by independent methods.

Although, harsh and lengthy dissociation methods may alter gene expression in cells ${ }^{2}$; effective isolation of cells is essential to obtain accurate representation of the cell types present. Since the bladder is a complex organ comprising multiple cell types, some populations such as urothelial or stromal cells may be relatively under-represented whereas other cell types such as fibroblasts exist within extracellular matrix and can be challenging to isolate. Dissociation becomes even more challenging if the bladder has undergone significant remodeling and fibrosis such as that observed in spinal cord injury ${ }^{3,4}$ or bladder outlet obstruction ${ }^{5,6}$.

Here, we describe an optimized tissue dissociation method for downstream single cell analysis in the spinal cord injured mouse bladder. Using flow cytometry, we compared four enzymatic digestion protocols for their ability to yield a single cell suspension, support cell viability and maintain the correct proportion of cell populations. Based on this analysis, we conclude that minimizing cell death, cellular aggregates, noncellular nucleic acids and potential inhibitors of downstream analysis are critical to achieving high quality data.

\section{Protocol}

The procedures were performed in strict accordance with the recommendations in the Guide for the Care and Use of Laboratory Animals of the National Institutes of Health. All experiments were approved by the Animal Care and Use Committee of Boston Children's Hospital.

NOTE: Mice were housed in an AAALAC-accredited animal facility with ad libitum access to food and water. Female mice at 8-12 weeks of age were used for these experiments. Given the nature of the injury, additional nutritional enrichment was provided to mice to ensure their wellbeing.

\section{Low-thoracic spinal cord transection in mice}

\section{Preparation before spinal cord transection}


NOTE: The surgical instruments required for this procedure are micro dissecting spring scissors, micro dissecting forceps, micro suturing needle driver, hemostats, and 7-0 polyglactin 910 sutures. Other surgical supplies required are surgical drapes, sterile sheets for surgical field, gauze sponges, cotton-tip applicators, and $1 \mathrm{~mL}$ syringes with $25 \mathrm{G}$ needles.

1. Autoclave the surgical instruments and supplies prior to the surgery.

2. Clean the surgical area and the heating pads with alcohol wipes. One heating pad will be used during the surgery and the other for the immediate postoperative period to maintain the animal's body temperature until regaining full activity.

3. Use magnifying loupes (2.5x or more) to perform the surgical procedure.

4. Switch on the heating pads, the light source, and the glass bead sterilizer to be ready for use during the procedure.

5. Open the surgical drapes and the instruments. Use sterile gloves to drape the surgical field and place the instruments in the surgical field.

\section{Preparation of the animals}

1. Bring the mice to the procedure room and also bring a clean cage for the spinal cord injured mice.

2. Administer anesthesia by placing the mouse in the induction chamber with isoflurane flow set at $3.0 \%$, oxygen flow at $1 \mathrm{~L} / \mathrm{min}$, and suction at $20 \mathrm{mmHg}$ until there is no paw-pinch response.

3. Immediately weigh the animal and then place the animal in the prone position on the heating pad.
4. Place the anesthetic cone snugly over the mouse's nose, switch the gas flow from the induction chamber to the nose cone, and set the isoflurane flow to $2 \%$ and oxygen flow to $1 \mathrm{~L} / \mathrm{min}$.

5. Confirm that the animal is adequately anesthetized with the absence of response to paw-pinch. Tape the animal limbs to the heating pad. Place a rolled piece of gauze sponge under the lower chest to elevate and flex open the lower thoracic vertebrae.

6. Apply ophthalmic lubricant to both eyes. Administer pain medication (Meloxicam, $10 \mathrm{mg} / \mathrm{kg}$, subcutaneously) and antibiotic (Enrofloxacin, $5 \mathrm{mg} /$ $\mathrm{kg}$, subcutaneously).

NOTE: End users should use the pain medication and antibiotics recommended by their local animal care and use committee.

7. Palpate the most prominent spinous process in the thoracic spine which typically corresponds with T13 spinous process ${ }^{7}$. Shave a longitudinal rectangle area on the back of the mouse from the lower neck to just below the most prominent spinous process (T13) and for $1 \mathrm{~cm}$ on each side of the midline.

\section{Surgical procedure}

1. Prep the shaved area with $10 \%$ povidone iodine solution and $70 \%$ ethanol three times alternatively in a circular fashion starting from the incision site working outward and then cover the animal with sterile $4 \times 4$ gauze sponge with a window in the center overlying the surgical field.

2. Make $1.5 \mathrm{~cm}$ incision in the midline of the back using fine scissors that ends at the most prominent spinous process (T13). The incision should include the skin and superficial fascia. Using scissors separate the 
skin and superficial fascia on either side to expose the spinous processes and the surrounding paraspinous muscles.

3. Using sharp and blunt dissection separate the muscles from the spinous processes and the laminae of T9, T10, and T11 vertebrae.

4. Sharply divide the interspinous ligaments between T9 and T10 and between T10 and T11 using fine scissors and then excise the spinous process of $\mathrm{T} 10$ and carefully perform T10 laminectomy bilaterally to expose the spinal cord. Ensure that the laminae are completely excised.

5. Transect the spinal cord using fine scissors. Minimal bleeding usually occurs at this point due to transection of the spinal cord vessels. Compress the bleeding area with a sterile cotton tipped applicator to achieve hemostasis. After confirming complete hemostasis, close the skin with 7-0 polyglactin 910 continuous sutures.

6. Administer $1 \mathrm{~mL}$ of saline solution subcutaneously to prevent postoperative dehydration.

\section{Postoperative care}

1. Place the animal on a heating pad until full recovery has occurred, then transfer to a cage for only spinal cord injured-mice.

2. Postoperative care includes observing and weighing the animals daily, and monitoring of the incision site for signs of infection for up to 7 days. Administer $1 \mathrm{~mL}$ saline solution, analgesic (meloxicam $10 \mathrm{mg} / \mathrm{kg}$ ), and antibiotic (enrofloxacin $5 \mathrm{mg} / \mathrm{kg}$ ) all subcutaneously daily for 3 days.
NOTE: End users should use the pain medication and antibiotics recommended by their local animal care and use committee.

3. Perform manual bladder expression (Credé maneuver) every $12 \mathrm{~h}$ until the animal is able to urinate on its own (usually in 10 to 14 days). Hold the animal with one hand and massage the lower abdomen with the other hand, then feel and gently compress the distended urinary bladder with the index finger and thumb. Gentle transient compression should alternate with relaxation. Following manual expression, wash the lower abdomen with tap water and dry gently with paper towel without excessive rubbing.

NOTE: A small size of the bladder before the beginning of the expression and wetting of the lower abdomen with urine are indications that the animal has gained the ability to void on its own.

4. To minimize weight loss, provide nutritional enrichment to mice in the form of nutritive gel and other nutritious treats (bacon softies, fruit crunchies and veggie bites) placed on the floor of the cage for easy access NOTE: In this study, mice were perfused and bladders procured at 8 weeks following SCI.

\section{Postoperative complications}

1. Minimize the potential for rupture of the bladder due to overzealous manual bladder expression by not fully expressing the bladder.

2. Prevent excoriation of the perineal skin from continuous exposure to urine dribbling from incompetent sphincter through washing of the perineal region with tap water. Minimize inflammation through application of triple antibiotic ointment. 
NOTE: Urethral obstruction due to blood clot during the period of transient hematuria or from semen coagulum due to retrograde ejaculation in male mice may occur following spinal cord injury. Complete urethral obstruction in male mice frequently culminates in bladder rupture and death. In our experience the frequency of urethral obstruction in male mice that led to death was $10 \%$.

\section{Perfusion and tissue procurement}

NOTE: For downstream analyses of certain cell types such as immune cells in peripheral tissues it is beneficial to remove blood by perfusion at the time of tissue harvest, as described below.

1. Administer anesthesia as mentioned in the surgical procedure (step 2.2) and confirm that the animal is adequately anesthetized with no forepaw-pinch response (the animal is paraplegic, therefore the hindlimbs have diminished sensation and the hindpaw-pinch response becomes irrelevant).

2. Place the animal in the supine position and swab the abdomen and chest with $70 \%$ ethanol to wet the fur to prevent it from getting into the operating site.

3. Perform a midline laparotomy from the pelvis to the diaphragm. Cut the diaphragm away from the ribs.

NOTE: Following this step, speed is important since the thoracic pressure differential no longer exists and the lungs cannot inflate, so the animal begins to suffocate.

4. Cut the thorax open along the ribs on the left and right side following the bone-cartilage border on a line parallel to the sternum, commencing at the diaphragm and proceeding as far as the first rib.
5. Place the complete anterior thoracic wall over the animal's head and fix it in this position using towel clamps. Do not cut off the anterior thoracic wall as this will cause severe bleeding from the two internal thoracic arteries.

6. Cut away the pericardium using fine scissors.

7. Connect a $23 \mathrm{G}$ needle to the perfusion apparatus, then insert it into the left ventricle and slowly into the aorta, taking care not to puncture it.

NOTE: The perfusion apparatus comprises a perfusion pump and $50 \mathrm{~mL}$ syringe connected to intravenous tubing.

8. Start the perfusion and quickly make a small cut with the tip of fine scissors in the right atrium for drainage. Take care not to introduce air bubbles during fluid infusion.

9. Perform perfusion with phosphate buffered saline (PBS) solution run at $15 \mathrm{~mL} / \mathrm{min}$. Perfusion is complete when drainage is clear and lightened color of the liver is achieved (Figure 1).

NOTE: The average time for perfusion was $3.5-4 \mathrm{~min}$. Inadequate perfusion is manifested as slow progression of blanching of tissues and usually is due to incorrect positioning of the needle in the left ventricle. Adjusting the needle and extending the duration of perfusion for 1 to 2 min will ensure adequate perfusion of tissues.

10. Discontinue the perfusion and dissect the bladder free from the vascular pedicles and urethra and place it in a microcentrifuge tube containing ice-cold Tyrode's solution

\section{Digestion of urinary bladder in control and spinal cord-injured mice}

NOTE: In order to formulate an efficient digestion mixture which is tailored for mouse urinary bladder we sought to adjust the unit of enzymes used to degrade the predominant 
extracellular matrix components such as collagens and hyaluronic acid. Therefore, we used publicly available RNA sequencing data generated by the Mouse ENCODE project (BioProject: PRJNA66167) to extract reads per kilobase per million (RPKM) and Tabula Muris ${ }^{8}$ for assessment of spatial expression within bladder. Collagens 1, 3 and 6 were the three most highly expressed genes among 42 different collagens (Figure 2A). The expression of those collagens and hyaluronan synthase 1 (Has1) were mostly observed in muscle cells and fibroblasts of the bladder wall (Figure 2B).

\section{Preparation of buffers and solutions}

1. Prepare sodium Tyrode solution as per Table $\mathbf{1}$ in a clean $500 \mathrm{~mL}$ bottle. Add $300 \mathrm{~mL}$ of $\mathrm{ddH}_{2} \mathrm{O}$. The solution is acidic after preparation. Adjust $\mathrm{pH}$ to 7.4 using $\mathrm{NaOH}$. Bring volume to $500 \mathrm{~mL}$ using double distilled $\mathrm{H}_{2} \mathrm{O}$, then aliquot and store at $-20^{\circ} \mathrm{C}$.

NOTE: This buffer maintains the $\mathrm{pH}$ and osmotic balance in the digestion buffer and provides the cells with water and essential inorganic ions. It contains magnesium, as well as glucose as an energy source. The potassium in the solution provides protective effects on electromechanical activity in the isolated cell solution. Powdered salts are hygroscopic and should be protected from moisture. The entire contents of the mix should be used immediately after preparation. Preparing a concentrated salt solution is not recommended as precipitates may form. Sterilization using filtration $(0.22 \mu \mathrm{m}$ filter $)$ can be performed if the cells are going to be cultured after analysis.

2. Prepare enzymatic digestion solution in a $15 \mathrm{~mL}$ conical tube by adding recommended volumes and amounts for each component (Table 2). Add sodium
Tyrode solution up to $2.5 \mathrm{~mL}$. Vortex thoroughly to dissolve.

NOTE: Papain is a sulfhydryl protease from Carica papaya latex. Papain has broad specificity and it will degrade most protein substrates ${ }^{9}$. Papain has proved less damaging and more effective than other proteases in cell dissociation protocols ${ }^{10}$. We provide details on the four dissociation protocols in Table 2; we observed protocol section 3 to support highest viability $(93 \%)$ of cell suspensions prepared from mouse bladder.

2. Dissociation procedure and preparation of cell suspension

1. Collect the bladder from mice post-perfusion.

2. Puncture the bladder to release contents, if any.

3. Add $100 \mu \mathrm{L}$ of Tyrode's solution to an empty $1.5 \mathrm{~mL}$ centrifuge tube and tare. Place the bladder in the tube and weigh again to determine an accurate bladder weight.

4. Place bladder on a $10 \mathrm{~cm}$ Petri dish on ice and add $100 \mu \mathrm{L}$ of Tyrode's solution for mincing.

5. Using surgical scissors, cut pieces as small as possible while minimizing the mincing time to no more than 2-3 min per bladder. If pooling bladder tissue from multiple animals, mince the bladders all at once.

6. Transfer the minced bladder tissue using a widebore pipet tip into $2.5 \mathrm{~mL}$ of digestion buffer for each bladder. Adjust the volume if multiple bladders are pooled. Incubate the tissue in digestion solution at 37 ${ }^{\circ} \mathrm{C}$ in an incubator on a nutator mixer for $40 \mathrm{~min}$. 
7. At the end of the incubation period, remove digestion tube from the incubator. Triturate (pipet up and down) digestion solution using a $5 \mathrm{~mL}$ pipette for $1 \mathrm{~min}$.

8. Centrifuge for $10 \mathrm{~min}$ at $350 \mathrm{xg}$ at $4{ }^{\circ} \mathrm{C}$. Remove the supernatant and resuspend the pellet in $1 \mathrm{~mL}$ of cell detachment solution. Place in a $37^{\circ} \mathrm{C}$ incubator on nutator mixer for $10 \mathrm{~min}$.

9. Centrifuge for $10 \mathrm{~min}$ at $350 \times g$ at $4{ }^{\circ} \mathrm{C}$. Remove the supernatant and resuspend the pellet in $1 \mathrm{~mL}$ of $\mathrm{RBC}$ lysis buffer (1x). Incubate for $1 \mathrm{~min}$.

10. Add $9 \mathrm{~mL}$ PBS to dilute the RBC buffer and stop the RBC lysis.

11. Pass cells through $70 \mu \mathrm{m}$ cell strainer into $50 \mathrm{~mL}$ conical tube, using the plunger from a syringe to lightly scrape the cell strainer to ensure full cell passage. Make sure to collect the liquid that passes through strainer but might be caught on underside of strainer.

12. Centrifuge for $10 \mathrm{~min}$ at $350 \mathrm{xg}$ at $4{ }^{\circ} \mathrm{C}$. Remove the supernatant and resuspend the pellet in $200 \mu \mathrm{L}$ of cell staining buffer (PBS with 2\% FBS).

13. Count the cells.

\section{Immunolabeling of specific cells for flow cytometry}

NOTE: To detect different types of cells in the bladder, we designed a multi-color flow cytometry panel. To perform compensation and devise an appropriate gating strategy, we include unstained and fluorescence minus one (FMO) controls. FMO controls are important to gate positive population, particularly when the positive fraction is dim. The staining procedure is as follows.

\section{Blocking FcyRII/III receptors on cells}

NOTE: We recommend to blocking nonspecific binding of monoclonal antibodies by pre-incubation of cells with monoclonal anti-Fc receptor antibodies, or recombinant Fc protein.

1. Wash the cells by centrifugation at $350 \times g$ for 5 min at $4{ }^{\circ} \mathrm{C}$ and add cell staining buffer.

2. Discard supernatant and block FcyRII/III receptors on cells to prevent non-specific antibody staining by adding CD16 and CD32 antibodies in cell staining buffer at dilution of $1: 100$.

3. Incubate on ice for $10 \mathrm{~min}$.

NOTE: There is no need to wash the cells; cells can be stained directly after this stage.

\section{Staining for FMOs}

NOTE: A Fluorescence Minus One (FMO) control is a tube of all fluorochromes used in the experiment that contains all the fluorochromes except one.

1. For example, if one has 4 different fluorochromes ( $\mathrm{A}, \mathrm{B} \mathrm{C}$ and $\mathrm{D}+$ Annexin $\mathrm{V}$ and propidium iodide $(\mathrm{PI})$ ), prepare the FMO tubes as following. FMO Tube 1: Antibodies conjugated with B, C, D fluorochromes + (Annexin V and PI); FMO Tube 2: Antibodies conjugated with A, C, D fluorochromes + (Annexin V and PI); FMO Tube 3: Antibodies conjugated with $A, B, C$ fluorochromes + (Annexin $\mathrm{V}$ and PI); FMO Tube 4: Antibodies conjugated with A, B, C, D fluorochromes + (Annexin V); FMO Tube 5: Antibodies conjugated with A, B, C, D fluorochromes $+(\mathrm{PI})$.

2. Consider the nature of the conjugated fluorochrome for the Annexin $V$ antibody.

\section{Staining the blocked cells with desired antibodies}


1. Incubate blocked cells with appropriate master mixes of fluorophore-conjugated antibodies against the desired proteins for $20 \mathrm{~min}$ on ice protected from light. Remember to include FMOs.

2. Wash cells with $1 \mathrm{~mL}$ of cell staining buffer added to each tube and then centrifuge again at $350 \times g$ for $5 \mathrm{~min}$ at $10^{\circ} \mathrm{C}$.

3. Discard the supernatant and resuspend the cell pellet in $200 \mu \mathrm{L}$ of cell staining buffer. Keep on ice until fluorescence data can be acquired using a flow cytometer.

\section{Apply Annexin V/ PI stain.}

1. Prepare a working solution of $\mathrm{PI}(100 \mu \mathrm{g} / \mathrm{mL})$ in $1 \mathrm{x}$ Annexin-binding buffer as described in the manufacturer's protocol for the dead cell apoptosis kit.

2. Determine the cell density and note the buffer and volume in which they are stored.

3. Centrifuge samples at $350 \times g$ for $5 \mathrm{~min}$, discard the supernatant and resuspend cells in $1 \mathrm{x}$ Annexin-binding buffer to a density of $\sim 1 \times 10^{6}$ cells $/ \mathrm{mL}$ in a volume of $100 \mu \mathrm{L}$.

4. Add FITC-Annexin $V(5 \mu L)$ and PI working solution $(1 \mu \mathrm{L})$ to each sample $(100 \mu \mathrm{L})$, as described in the manufacturer's protocol, and incubate at room temperature for $15 \mathrm{~min}$.

5. Add $400 \mu \mathrm{L}$ of $1 \mathrm{x}$ Annexin-binding buffer to samples, mix by inversion and keep on ice until flow cytometry.

\section{FACS calibration}

\section{Flow cytometry and purity control}

1. Start flow cytometry analysis by measuring the unstained cells to delineate cell morphology and the troughs of the fluorochromes.

2. Adjust the side scatter (SSC) and forward scatter (FSC) by modifying the voltages of each fluorescence parameter. Measure the fluorescence emission at $530 \mathrm{~nm}$ (Annexin V) and $>575 \mathrm{~nm}(\mathrm{Pl})$.

3. Define the negative population in the first decade by using the grids on each dot plot. Place each FMO control in the cytometer and correct the spectral overlap until the negative and positive population medians are aligned.

4. Measure 100,000 events. Measure cells stained with specific markers and create gates for cell populations of interest.

\section{Data analysis}

1. Collect the data from the flow cytometer. Open the software to visualize the workspace for analysis.

\section{Creating a workspace}

1. Import FCS files by dragging them into the workspace. Files will be visible in the sample and group section of the workspace. Double-click in the sample name to open the file.

2. Use side scatter area (SSC-A) for the $y$-axis and forward scatter area (FSC-A) for the $x$-axis (Figure 3A). Click the icon for polygon gating.

3. Create a gate around the cell population of interest in the dot plot by clicking to make a gate node and then continue clicking around the cell population until complete; double click to close the gate. 
4. Name the gate according to the population captured (e.g., "All cells") and click OK.

NOTE: Double clicking within the "All cells" gate will open a new graph window showing only the events contained in "All cells".

5. Adjust the y-axis of the new dot plot to SSC-H (side scatter height) by clicking in the black arrow and select to change the y-axis.

NOTE: This gates for single cells (singlets) and excludes doublets or larger aggregates (Figure 3B). Since single cells have proportional width and length, they should be represented as a population on the diagonal. Cells falling outside of this diagonal gate are doublets or larger aggregates.

6. Double click on the gate to analyze necrotic (PIpositive), early apoptotic (Annexin V-positive, PInegative) and late apoptotic (Annexin V-positive, PI-negative) cells (Figure 3C).

7. Label the $x$-axis as Annexin $V$ and the $y$-axis as PI. NOTE: In some cases, where signal intensity is low, cell populations may appear to have negative fluorescence values, as a result of correction for background. In this case, it is recommended to perform a bi-exponential transformation. To do this, click on the $\mathbf{T}$ next to the $y$-axis and choose Customize Axis. In the new window change the scale to bi-exponential (Biex), add negative values to the axes by increasing the width basis and click Apply. This will improve the resolution of events with low signal intensity.
8. Show data as a count plot. Use the Option tab just below the $x$-axis and select counter plot from the menu.

9. Draw a Quat gate on the plot to define 4 discrete target populations.

10. Click at the top of the window to open the layout editor, by clicking in Layout editor and drag populations into each separate area.

11. Place plots into the layout editor by dragging and dropping populations from the workspace into the layout editor window.

\section{Visualizing using histograms}

1. Choose histogram from the Options tab.

2. Apply a gate to select Annexin V-positive cells; alternatively, positive and negative populations can be defined by using the bisector tool. The sample section must now show the different populations that have been form and their hierarchy.

3. To compare samples, drag all the histograms on top of each other; right click on the histogram and from histogram choose Stagger Offset.

\section{Add statistical analyses}

1. Open the Statistic tab by double clicking on the population of interest. Select the function to apply and the parameter involved.

2. Repeat with other populations, by dragging the Sigma icon into the population name.

3. Apply the analysis to all samples by selecting the gating strategy from the sample of interest and 
dragging it into the group defined by the marker of interest, e.g. Annexin V.

4. Create gates in the FMO control samples and define negative and positive populations; this gating strategy will be applied to the entire experiment (Figure 3D-F).

NOTE: Check each sample individually to ensure gating is correct and modify where necessary.

5. If cells have been stained with marker antibody (e.g., CD45) use the corresponding FMO and gate accordingly.

6. To export the layout, click on File | Export Image | Select file format (e.g., jpg, pdf).

7. Click Create Table to open a window with the final version of the table.

8. Export the table by selecting File | Save As | Filename.

\section{Representative Results}

\section{Surgical procedure}

The success of thoracic spinal cord transection is determined by assessment of a number of parameters, the most obvious of which is hindlimb paralysis. The animal moves only using its forelimbs, dragging its hindlimbs. Otherwise activity levels, including feeding, grooming and alertness are typically normal. In addition, animals lose volitional bladder control resulting in the need for manual bladder expression by the investigator every $12 \mathrm{~h}$ until reflex voiding returns at 10 to 14 days after injury. Following euthanasia, additional signs of the success of the injury relate primarily to the increase in bladder-to-body weight ratio, indicative of tissue remodeling. Histological analysis reveals hyperplasia within both the urothelial and smooth muscle compartments ${ }^{3}$.

\section{Preparation of single cell suspension}

Using publicly available expression data, the enrichment of bladder tissue for extracellular matrix proteins was determined (Figure 2) and used to inform the formulation of the digestion mix. Since collagens are key components of the bladder wall ${ }^{11,12}$, first we sought to determine the most abundant collagen(s) in the mouse bladder using RNA profiling data sets generated by the Mouse ENCODE project $^{13}$. Our analysis showed that Collagen $1 \mathrm{~A} 1$, collagen $3 \mathrm{~A} 1$, collagen $1 \mathrm{~A} 2$ and collagen $6 \mathrm{~A} 1$ are most abundant collagen types within mouse bladder (Figure 2A). We also used the Tabula Muris (a compendium of single cell transcriptome data from mouse (Mus musculus)) $)^{8}$ to determine the mRNA expression level of collagens 1, 3, 6 and hyaluronan. The data allow for direct and controlled comparison of gene expression in cell types shared between tissues. This analysis revealed that the expression of these extracellular matrix components is more prevalent in the mesenchymal cell types rather than urothelium (Figure 2B).

\section{Effect of dissociation on viability of isolated cells from} bladder

Flow cytometry analysis demonstrated that enzymatic digestion using the 4 different protocols yielded viability of $83 \%, 86 \%, 93 \%$ or $90 \%$, respectively. Thus, protocol section 3 was deemed most valuable for preservation of cell viability. We also observed that approximately $4 \%$ of the cells were necrotic $\left(\mathrm{PI}^{+} /\right.$Annexin $\left.\mathrm{V}^{-}\right)$(Figure 4A). These observations emphasize the efficiency of the digestion protocol and the ensuing benefit on cell viability.

\section{Effect of spinal cord injury on different populations of cells in the bladder}

We observed a significant increase in the total cell number in bladders of $\mathrm{SCl}$ mice compared to controls. The pattern 
of the dot plots obtained from $\mathrm{SCl}$ bladders was also slightly different consistent with ongoing organ remodeling due to spinal cord injury (Figure 4B: first column). Compared to controls, the bladders from $\mathrm{SCl}$ animals displayed a significant increase in CD45-positive cells.
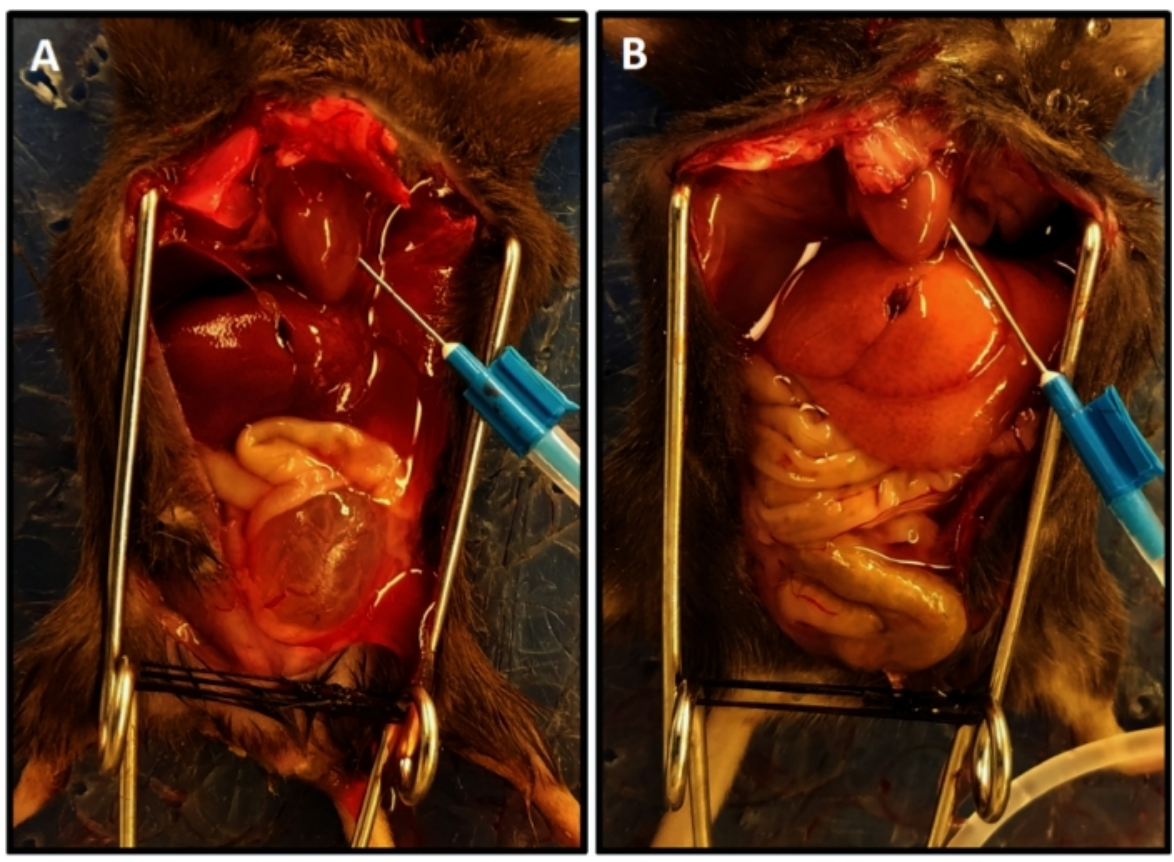

Figure 1: Representative perfusion completion with lightened color of the liver. (A) Demonstrates the liver color at the beginning of the perfusion. (B) Shows the lightened liver color at the end of perfusion. The mouse in (A) had spinal cord transection two weeks prior to perfusion resulting in bladder hypertrophy and its protrusion out of the pelvis unlike the mouse in (B) that did not have spinal cord injury; in this case the bladder is small and hidden in the pelvis. Please click here to view a larger version of this figure. 

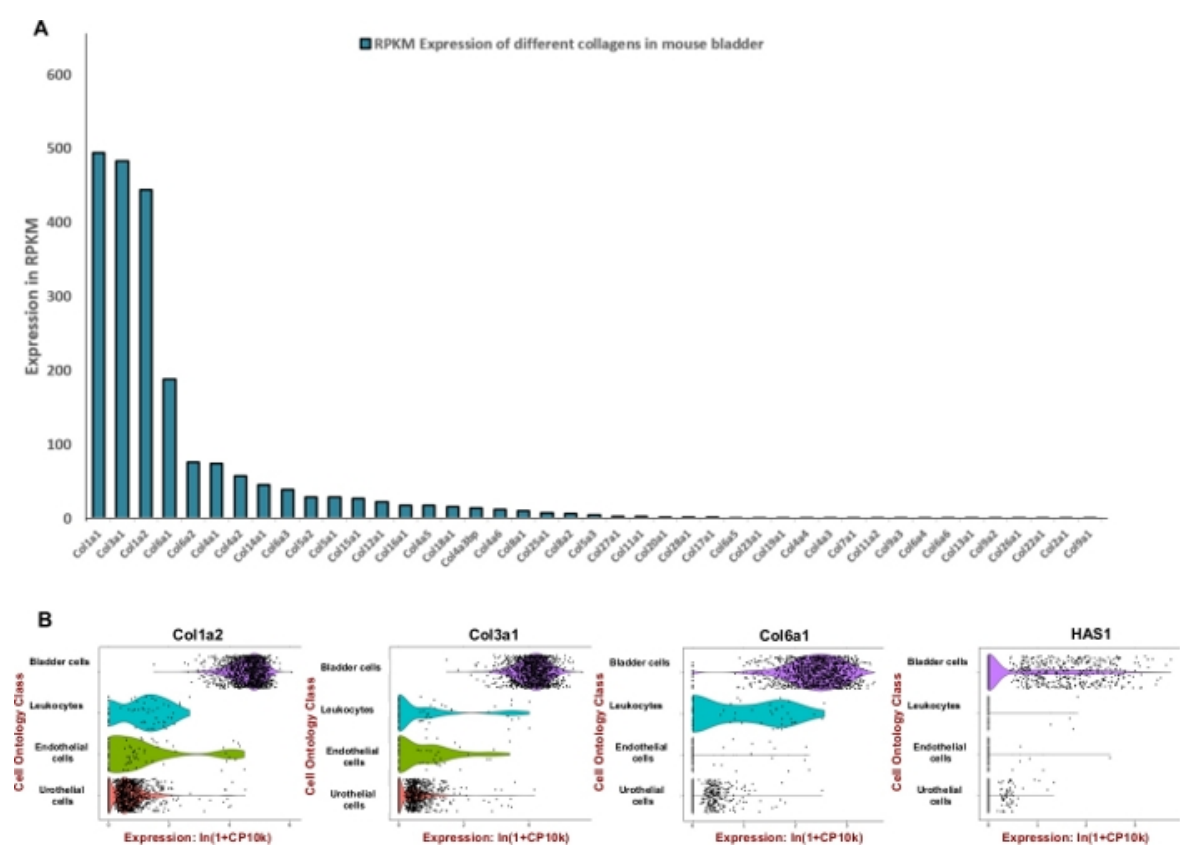

Figure 2: Transcriptomic expression of extracellular matrix (ECM) components in the mouse bladder. (A) Bar chart of 43 different collagen types. The expression is stated by Reads Per Kilobase of transcript, per million mapped reads (RPKM) (data is collected from BioProject: PRJNA66167) ${ }^{14}$. (B) Violin plots of gene expression in cell types obtained from microfluidic droplet-based 3'-end counting in a pool of male and female dissociated urinary bladder samples (male and females). Counts were log-normalized for each cell using the natural logarithm of $1+$ counts per million $\ln (C P M+1)^{8}$. A pseudocount of 1 CPM was added before taking logarithms. Please click here to view a larger version of this figure. 

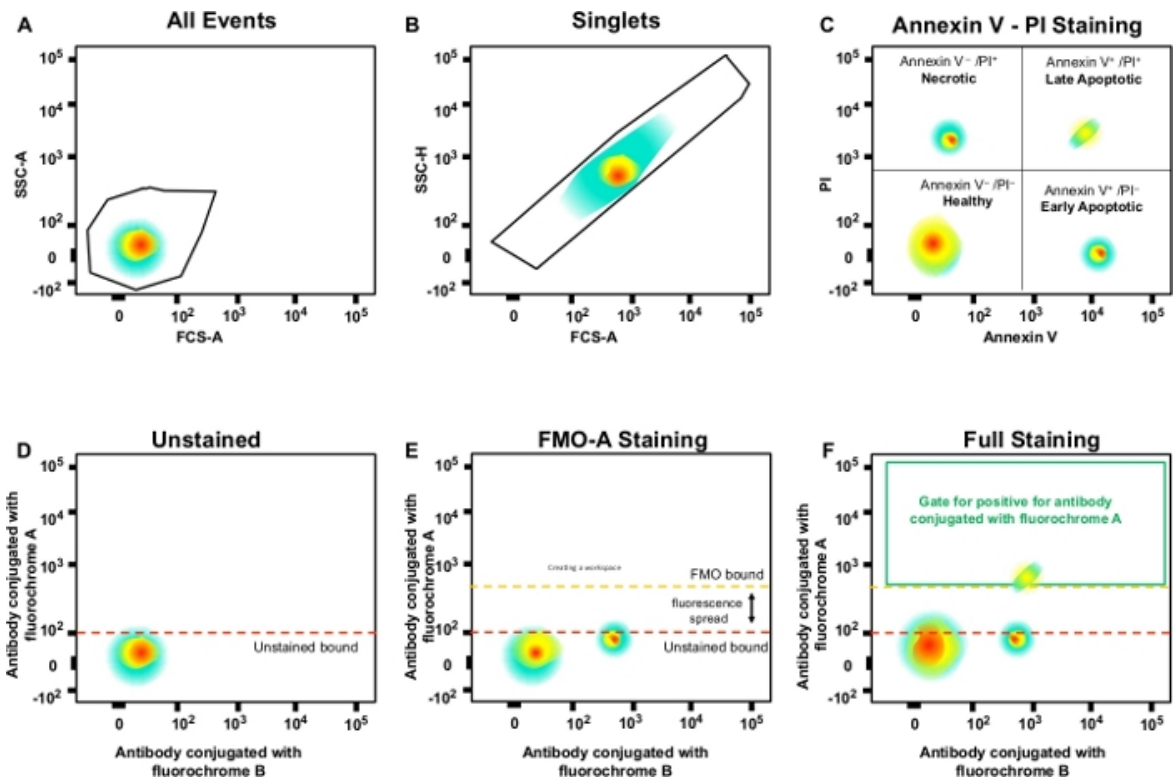

Figure 3: Gating strategy and FMO controls to determine fluorescence spread. (A) Selection of cell population. (B)

Gating strategy for singlets. (C) Gating for necrotic, and early and late apoptotic cells using PI and Annexin V antibody. (D-F) A schematic dot plot of multicolor flow cytometry (e.g., antibodies conjugated with A, B, C, D fluorochromes $+($ Annexin $V$ and $\mathrm{PI})$. This shows the fluorescence spread into the antibody with fluorochrome A channel shown by the FMO control compared to an unstained control. Orange dotted line represents FMO gating boundary compared to unstained boundary in red. Please click here to view a larger version of this figure. 

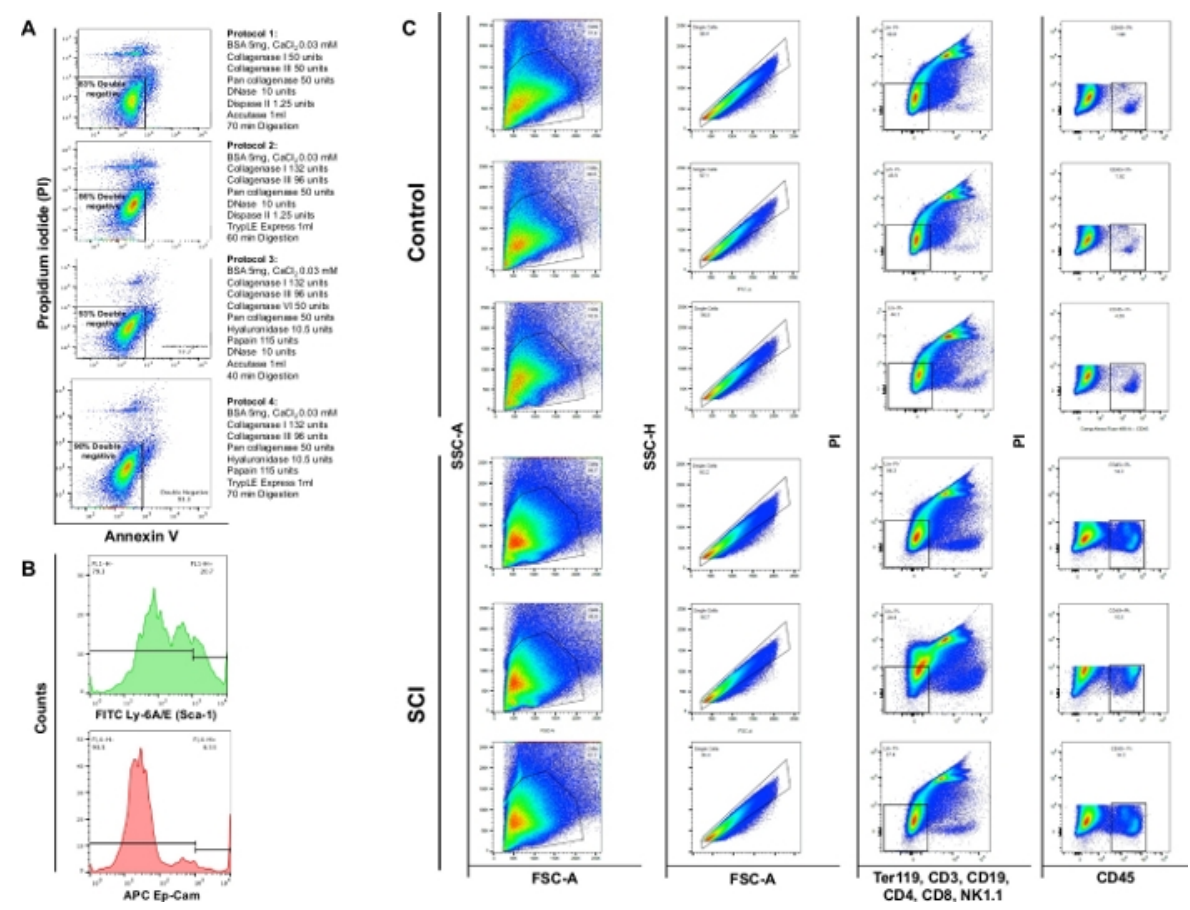

Figure 4: Flow cytometry of different cell types in bladder. (A) Annexin V/PI double staining flow charts. The different combinations of enzymes and chemical used for each protocol are represented in front of the corresponding viability plot. These data demonstrate highest viability was obtained with protocol section 3. (B) Representative histograms illustrating the intensity of Ly-6A/E (Sca-1) and CD326 (Ep-CAM) detected in single channels. (C) Effect of SCl on cell population of the mouse bladder. Upper panel shows results of staining on three dissociated bladders obtained from control non-surgical mice and lower panel shows the results of staining on three animals with $\mathrm{SCl}$. The first column is the total cell population. The second column shows the singlet gating selection. The third column shows the subpopulation of live cells that are negative for B-cells, T-cells and NK cells. The fourth column shows the staining for live cells positive for CD45. Please click here to view a larger version of this figure. 


\begin{tabular}{|c|c|c|}
\hline Component & Amount (for $\mathbf{5 0 0} \mathbf{~ m L}$ ) & Molarity \\
\hline $\mathrm{NaCl}$ & $4.091 \mathrm{~g}$ & $5 \mathrm{mM}$ \\
\hline $\mathrm{KCl}$ & $0.186 \mathrm{~g}$ & $1 \mathrm{mM}$ \\
\hline $\mathrm{MgCl} 2$ & $0.0476 \mathrm{~g}$ & $10 \mathrm{mM}$ \\
\hline D-Glucose & $0.9 \mathrm{~g}$ & $10 \mathrm{mM}$ \\
\hline HEPES & $1.19 \mathrm{~g}$ & 2 \\
\hline
\end{tabular}

Table 1: Components for preparation of Tyrode's solution. The indicated components are for preparation of $500 \mathrm{~mL}$ Tyrode's solution. 


\begin{tabular}{|c|c|c|c|c|c|}
\hline Component & Amount & Protocol section 1 & Protocol section 2 & Protocol section 3 & Protocol section 4 \\
\hline BSA & $5 \mathrm{mg}$ & yes & yes & yes & yes \\
\hline $\mathrm{CaCl}_{2}$ & $0.03 \mathrm{mM}$ & yes & yes & yes & yes \\
\hline Collagenase Type I & 132.5 units & yes & yes & yes & yes \\
\hline $\begin{array}{c}\text { Collagenase } \\
\text { Type III }\end{array}$ & 96.4 units & yes & yes & yes & yes \\
\hline $\begin{array}{c}\text { Collagenase } \\
\text { Type VI }\end{array}$ & 50 units & - & - & yes & - \\
\hline DNase & 10 units & yes & yes & yes & - \\
\hline Papain & 115 units & - & - & yes & yes \\
\hline Pan Collagenase & 50 units & - & - & yes & yes \\
\hline Hyaluronidase & 10.5 units & - & - & yes & yes \\
\hline Dispase II & 1.25 units & yes & yes & - & - \\
\hline $\begin{array}{c}\text { Cell dissociation } \\
\text { solution }\end{array}$ & $1 \mathrm{~mL}$ & yes & - & yes & - \\
\hline $\begin{array}{c}\text { Recombinant } \\
\text { enzyme }\end{array}$ & $1 \mathrm{~mL}$ & - & yes & - & yes \\
\hline
\end{tabular}

Table 2: Components for preparation of digestion buffer. The indicated components are for preparation of $2.5 \mathrm{~mL}$ digestion mix $\left(1 \mathrm{U}\right.$ catalyzes the hydrolysis of $1 \mu \mathrm{mol}$ a substrate per minute at $37^{\circ} \mathrm{C}$. Refer to the product data sheet for definition of unit of each enzyme).

\section{Discussion}

The mouse spinal cord injury model described here provides a reproducible method to create a functional bladder outlet obstruction due to loss of coordination between bladder contraction and external urethral sphincter relaxation. This in turn evokes profound remodeling of the bladder wall as early as 2 weeks after injury characterized by expansion of urothelial and smooth muscle compartments. Critical steps in implementation of the $\mathrm{SCl}$ model in rodents include (i) rigorous attention to manual bladder expression during the period of spinal shock that ensues for 10-14 days after injury; (ii) nutritional enrichment to minimize weight loss; and (iii) mitigation of the potential for urine scalding particularly for experiments that extend beyond the return of reflex voiding. Limitations of the model include the potential for urethral occlusion in mice from blood clots during the period of transient hematuria, and additionally in male mice from 
semen coagulum following retrograde ejaculation following surgery.

The tissue dissociation approach described here illustrates the importance of considering structural changes in tissues that arise from the experimental insult, in this case significant tissue remodeling following SCI that may influence downstream analyses. With the increase in single cell analyses it is critical to ensure that differences observed in gene expression are not simply a result of dissociationinduced perturbations, but are truly representative of underlying biological changes relevant to the disease model. The use of publicly available expression data allowed us to modify the formulation of digestion buffers to ensure effective digestion of extracellular matrix while maximizing viability. Additional modifications that could be considered in future applications include the addition of actinomycin $D$, to halt transcription of immediate early genes that are sensitive to the dissociation protocol ${ }^{15}$.

Pipetting technique is crucial when dissociating tissue or transferring cells that are already in suspension. To reduce physical damage to cells from shearing forces, it is important to pipette gently and slowly during cell resuspension. It is generally recommended to use wide-bore pipette tips. If using standard tips, it is particularly important to pipette cell suspensions gently to avoid shear forces that would otherwise damage cells. Using cell strainers is unavoidable in this protocol, however, the cell concentration can decline by $20 \%$ or more, accompanied by a volume loss of $100 \mu \mathrm{L}$ or more. We recommend that cell concentration be determined after straining to ensure a precise cell count.

In flow cytometry, FMO controls provide a measure of background due to bleed-through of signal from overlapping emission peaks. They are not a measure of nonspecific antibody binding, or background staining that may be present when an antibody is included in that channel. To account for the nonspecific antibody binding, one has to include appropriate isotype controls; for the background staining, one needs to include negative controls. Taken together, these controls ensure accurate measurement of cell populations.

\section{Disclosures}

No conflicts of interest declared.

\section{Acknowledgments}

This work was supported by grants from the National Institutes of Health (R01 DK077195 to R.M.A, R01 DK104641 to R.M.A and D.R.B). We acknowledge valuable input from Dr. Stuart Orkin in the Division of Hematology/Oncology, Boston Children's Hospital, Department of Pediatrics, Harvard Medical School and the Dana-Farber Cancer Institute. We also acknowledge support from Kyle Costa in post-operative care of mice, Mary Taglienti and Dr. Habiballah Shojaeisaadi (Dr. Yang Shi Laboratory, Dept. of Pediatrics, Division of Newborn Medicine, Dept. of Pediatrics, Division of Newborn Medicine, Boston Children's Hospital, Harvard Medical School) for technical assistance and helpful discussions.

\section{References}

1. Grange, C. et al. Phenotypic characterization and functional analysis of human tumor immune infiltration after mechanical and enzymatic disaggregation. Journal of Immunological Methods. 372 (1-2), 119-126 (2011).

2. van den Brink, S. C. et al. Single-cell sequencing reveals dissociation-induced gene expression in tissue subpopulations. Nature Methods. 14 (10), 935-936 (2017). 
3. Seth, A. et al. The impact of discrete modes of spinal cord injury on bladder muscle contractility. BMC Urology. 13 24 (2013).

4. Doyle, C. et al. Inosine attenuates spontaneous activity in the rat neurogenic bladder through an A2B pathway. Scientific Reports. 744416 (2017).

5. Gheinani, A. H. et al. Characterization of miRNAregulated networks, hubs of signaling, and biomarkers in obstruction-induced bladder dysfunction. JCI Insight. 2 (2), e89560 (2017).

6. Gheinani, A. H. et al. Concordant miRNA and mRNA expression profiles in humans and mice with bladder outlet obstruction. American Journal of Clinical and Experimental Urology. 6 (6), 219-233 (2018).

7. Krishna, V. et al. A contusion model of severe spinal cord injury in rats. Journal of Visualized Experiments. (78) (2013).

8. The Tabula Muris Consortium. et al. Single-cell transcriptomics of 20 mouse organs creates a Tabula Muris. Nature. 562 (7727), 367-372 (2018).

9. Gray, C. J., Boukouvalas, J., Szawelski, R. J.,Wharton, C. W. Benzyloxycarbonylphenylalanylcitrulline $p$-nitroanilide as a substrate for papain and other plant cysteine proteinases. Biochemical Journal. 219 (1), 325-328 (1984).

10. Feodorova, Y., Koch, M., Bultman, S., Michalakis, S., Solovei, I. Quick and reliable method for retina dissociation and separation of rod photoreceptor perikarya from adult mice. MethodsX. 2 39-46 (2015).

11. Aitken, K. J., Bagli, D. J. The bladder extracellular matrix. Part I: architecture, development and disease. Nature Reviews Urology. 6 (11), 596-611 (2009).
12. Aitken, K. J., Bagli, D. J. The bladder extracellular matrix. Part II: regenerative applications. Nature Reviews Urology. 6 (11), 612-621 (2009).

13. The ENCODE Project Consortium. An integrated encyclopedia of DNA elements in the human genome. Nature. 489 (7414), 57-74 (2012).

14. Yue, F. et al. A comparative encyclopedia of DNA elements in the mouse genome. Nature. 515 (7527), 355-364 (2014).

15. Wu, Y. E., Pan, L., Zuo, Y., Li, X., Hong, W. Detecting Activated Cell Populations Using Single-Cell RNA-Seq. Neuron. 96 (2), 313-329 e316 (2017). 